DESY 95-098

Fermilab-Pub-95/131-T

\title{
Cross Sections for Charm Production in ep Collisions: Massive versus Massless Scheme
}

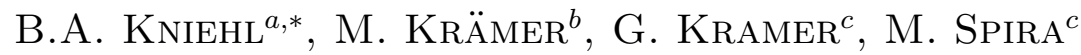 \\ ${ }^{a}$ Fermi National Accelerator Laboratory, Batavia, IL 60510, USA \\ ${ }^{b}$ Deutsches Elektronen-Synchrotron DESY, D-22603 Hamburg, Germany \\ ${ }^{c}$ II. Institut für theoretische Physik ${ }^{\dagger}$, Universität Hamburg, D-22761 Hamburg, Germany
}

\begin{abstract}
The next-to-leading order inclusive cross section for large- $p_{\perp}$ photoproduction of charm quarks at HERA is calculated in two different approaches. In the first approach the charm quarks are treated as massive objects which are strictly external to the proton and the photon while in the second approach the charm mass is neglected and the $c$ quark is assumed to be one of the active flavours in the proton and photon structure functions. We present single-inclusive distributions in transverse momentum and rapidity including direct and resolved photons. The cross section in the massless approach is found to be significantly larger than in the massive scheme. The deviation originates from several contributions which are disentangled. We argue that large- $p_{\perp}$ photoproduction of charm quarks at HERA will be sensitive to the charm content of the photon structure function.
\end{abstract}

$\dagger$ Supported by Bundesministerium für Forschung und Technologie, Bonn, Germany, Contract 056 HH 93P (5) and EEC Program "Human Capital and Mobility" through Network "Physics at High Energy Colliders" under Contract CHRX-CT93-0357 (DG12 COMA).

* Permanent address: Max-Planck-Institut für Physik, Föhringer Ring 6, 80805 Munich, Germany. 


\section{Introduction}

Charm production in high energy ep collisions at HERA is dominated by photoproduction events where the electron is scattered by a small angle producing photons of almost zero virtuality $\left(Q^{2} \simeq 0\right.$ ). In leading order (LO) QCD the main process is photon-gluon fusion where the photon interacts directly with the gluon from the proton producing a $c \bar{c}$ pair in the final state $(\gamma+g \rightarrow c+\bar{c})$. Besides the direct photoproduction channel charm production at HERA can proceed also via the resolved photoproduction process where the photon behaves as a source of partons which interact with the partons in the proton, as for example in $g+g \rightarrow c+\bar{c}$. It is known that for the photoproduction of light quarks $q$ (i.e. $q=u, d$ and $s$ ) and gluons the cross section of the resolved process is much larger than the cross section for direct photoproduction 迎仼. Only at rather large transverse momenta, $p_{\perp} \gtrsim 30 \mathrm{GeV}$, the cross sections for the two types of processes are comparable. This has been confirmed in various ways by measurements of the ZEUS [5] and H1 [6] collaborations at HERA. Actually a recent comparison of the ZEUS experimental data for the inclusive onejet cross section [7] with the next-to-leading order (NLO) predictions superimposing resolved and direct photoproduction shows satisfactory agreement in absolute normalization, $p_{\perp}$ and rapidity dependences [8]. These predictions are meant to be valid only for large enough $p_{\perp}$, i.e. $p_{\perp} \gtrsim 5 \mathrm{GeV}$, so that soft processes, which can not be calculated in perturbative QCD, are negligible. In the calculations the authors assume four active flavours, $q=u, d, s$ and $c$, which are taken to be massless. This means that also charm quarks are produced and counted as jets. Of course, the $c$ quark is also an ingoing parton originating either from the proton or the photon in the case of the resolved contribution. To neglect the charm mass $m$ in these calculations is a reasonable approximation since in large- $p_{\perp}$ jet production the effective scale is $p_{\perp}$ which is much larger than $m \simeq 1.5 \mathrm{GeV}$. The same approach is followed for predicting inclusive cross sections for the photoproduction of light hadrons, like $\pi$ 's, $K$ 's etc., when the produced massless $c$ quarks fragment into the light hadrons similarly as the produced $u, d, s$ quarks and the gluons [9]. In all of these theoretical investigations the $c$ quark is one of the massless active flavours in the photon and proton structure functions. In the following we shall refer to this as the massless charm scheme, which we assume to be valid in the region of large transverse momenta $p_{\perp} \gg m$. In this scheme the small $p_{\perp}$ region is not calculable. The cross section diverges in the limit $p_{\perp} \rightarrow 0$ and total production rates can not be predicted.

In the massive charm scheme which has been adopted by many authors (for a review see [10]) the charm mass, $m \gg \Lambda_{\mathrm{QCD}}$, acts as a cutoff and sets the scale for the perturbative calculations. Similarly to the case of large- $p_{\perp}$ jet production the cross section factorizes into a partonic hard scattering cross section multiplied by light quark and gluon densities [11. Inherent in this factorization is the notion that the only quarks in the hadron and the photon are the light ones. Thus in the massive charm scheme the number of active flavours in the initial state is equal to $n_{f}=3$ and the massive $c$ quark appears only in the final state. This approach has the advantage that not only various distributions, like in rapidity and/or transverse momentum, can be predicted but also the total cross section. In LO direct production is described by the partonic reaction $\gamma+g \rightarrow c+\bar{c}$ while the resolved contribution involves the channels $q+\bar{q} \rightarrow c+\bar{c}$ and $g+g \rightarrow c+\bar{c}$, where $q(\bar{q})$ are light (massless) quarks. NLO corrections have been calculated to these processes and found to be substantial [12,13]. A comparison between the theoretical NLO results and experimental data on photoproduction of charm quarks in low-energy $\gamma p$ and $\gamma \gamma$ collisions has shown reasonable agreement [14. Recently, the total charm production cross section at HERA has been measured [15] and 
found to be well described by the prediction of Frixione et al. [16. Depending on the choice of the photon structure function the resolved cross section seems to contribute only a fraction to the total charm production cross section [16].

One might expect that the massive approach is reasonable only in those kinematical regions where the mass $m$ and any other energy scale like $p_{\perp}$ are approximately of the same magnitude and significantly larger than $\Lambda_{\mathrm{QCD}}$. Under these circumstances the charm mass is used as the scale of $\alpha_{s}$ and the quark and gluon densities of the photon and the proton. In next-to-leading order terms $\sim \alpha_{s}\left(\mu^{2}\right) \ln \left(p_{\perp}^{2} / m^{2}\right)$ arise from collinear emission of gluons by a heavy quark at large transverse momentum or from almost collinear branching of gluons or photons into heavy quark pairs. These terms are not expected to affect the total production rates, but they might spoil the convergence of the perturbation series and cause large scale dependences of the NLO result at $p_{\perp} \gg m$. In the massive approach the prediction of differential cross sections is thus limited to a rather small range of $p_{\perp} \sim m$. The proper procedure for $p_{\perp} \gg m$ is to absorb the terms proportional to $\ln \left(p_{\perp}^{2} / m^{2}\right)$ into the charm distribution functions of the incoming photon and proton and into the fragmentation function of $c$ quarks into charmed hadrons. Of course, to do this absorption one needs a charm contribution in the structure function in the first place. An alternative way of making predictions at large $p_{\perp}$ is to treat the charm quarks as massless partons from the start. The mass singularities of the form $\ln \left(p_{\perp}^{2} / m^{2}\right)$ are then absorbed into structure and fragmentation functions in the same way as for the light $u, d, s$ quarks. We expect this massless approach to be better suited for the calculation of the differential $p_{\perp}$ distributions up to NLO in the region $p_{\perp} \gg m$. Then the problem arises how to proceed in the intermediate range where $p_{\perp}>m$.

In order to investigate the region where $p_{\perp}>m$ we have calculated the differential cross section $\mathrm{d}^{2} \sigma / \mathrm{d} y \mathrm{~d} p_{\perp}^{2}$ as a function of $p_{\perp}$ with the rapidity $y$ integrated over the region $|y| \leq 1.5$. We shall compare the results in the two approaches: (a) the massive charm approach with $m=1.5 \mathrm{GeV}$, in which we have computed the cross section for open charm production and (b) the massless approach, where we have evaluated the same differential cross sections for inclusive charmed particle production. In both calculations we include direct and resolved processes and go up to NLO where necessary. The massive calculation is based on the work presented in [13] while the massless predictions use the results obtained in [17]. In the second approach we need the fragmentation function of the $c$ quark into charmed hadrons. This is approximated by $\delta(1-z)$ where $z=p_{D} / p_{c}$ is the scaled momentum of the charmed hadron, meson or baryon. With this choice the LO results in the massive scheme approach the LO massless results in the limit $m \rightarrow 0$ if we restrict ourselves to the same parton subprocesses. They must differ, however, in NLO where the limit $m \rightarrow 0$ is not possible due to the unabsorbed mass singular $\ln \left(p_{\perp}^{2} / m^{2}\right)$ terms. Of course it is no problem to incorporate more realistic $c$ fragmentation functions in both schemes but the choice above should be sufficient to see the essential differences of the two approaches. A similar study of the production of large- $p_{\perp}$ hadrons containing bottom quarks in $p \bar{p}$ collisions has been performed by Cacciari and Greco [18]. 


\section{Comparison of Results}

We have calculated the cross section $\mathrm{d}^{2} \sigma / \mathrm{d} y \mathrm{~d} p_{\perp}^{2}$ in the HERA laboratory frame with $E_{p}=$ $820 \mathrm{GeV}$ protons colliding with $E_{e}=26.7 \mathrm{GeV}$ electrons travelling in the $(+z)$ direction $(y>0)$. The virtual photon spectrum is described in the Weizsäcker-Williams approximation with the formula

$$
f_{\gamma / e}(x)=\frac{\alpha}{2 \pi}\left[\frac{1+(1-x)^{2}}{x} \ln \frac{Q_{\max }^{2}}{Q_{\min }^{2}}+2 m_{e}^{2} x\left(\frac{1}{Q_{\max }^{2}}-\frac{1}{Q_{\min }^{2}}\right)\right]
$$

with $Q_{\max }^{2}=4 \mathrm{GeV}^{2}, Q_{\min }^{2}=m_{e}^{2} x^{2} /(1-x)$ and $x=E_{\gamma} / E_{e}$ in the interval $0.15<x<0.86$ as in [15] which corresponds to $\gamma p$ c.m. energies of $115 \mathrm{GeV}<W<275 \mathrm{GeV}, m_{e}$ is the electron mass. Since the average $Q^{2} \simeq 10^{-4} \mathrm{GeV}^{2}$ is very small, the photons are essentially on-shell, so that the $Q^{2}$ dependence of $\sigma_{\gamma p}$ and the longitudinal contribution in (11) can be neglected. For the proton structure function we use the $\operatorname{MRS}(\mathrm{G})$ set [19] which describes well the proton structure at small $x$ and is adjusted in the intermediate $x$ range. In Fig.1a the results for the cross section $\mathrm{d}^{2} \sigma / \mathrm{d} y \mathrm{~d} p_{\perp}^{2}$ averaged over the rapidity range $|y|<1.5$ are presented for the direct contribution in the massless and in the massive scheme. Both LO and NLO predictions are shown. In the LO cross section the same NLO parton structure function MRS $(\mathrm{G})$ has been adopted, only the parton-parton scattering cross sections are evaluated in LO. The two-loop formula for $\alpha_{s}$ is used with the $\Lambda$ value taken from the $\operatorname{MRS}(\mathrm{G})$ fit: $\Lambda_{\overline{\mathrm{MS}}}^{(4)}=255 \mathrm{MeV}$. Both NLO calculations have been performed in the $\overline{\mathrm{MS}}$ factorization and renormalization schemes. The corresponding scales have been set to $\mu=\sqrt{p_{\perp}^{2}+m^{2}}$. It is clear that in the massive scheme only three flavours are active in the initial state and in the evaluation of $\alpha_{s}$ whereas in the massless scheme also the charm distribution in the proton contributes to $c(\bar{c})$ production and $\alpha_{s}$ is calculated using four active flavours. We observe that the predictions in the massless and massive scheme are very similar. In LO there is some difference, the LO massless cross section is approximately $20 \%$ larger than the massive cross section over the whole range of $p_{\perp}$ between 3 and $15 \mathrm{GeV}$. This small difference originates from the additional charm contribution in the proton and the value of $\alpha_{s}$. In NLO the direct contributions of the massless and massive theories yield almost identical results for the $p_{\perp}$ distribution in the range $3<p_{\perp}<15 \mathrm{GeV}$. We have checked that this is true also for higher values of the transverse momentum up to $p_{\perp} \sim 30 \mathrm{GeV}$.

The situation is completely changed for the resolved part of the $c / \bar{c}$ production. This contribution has been calculated with the NLO photon structure function of GRV [20] in the $\overline{\mathrm{MS}}$ scheme. The results for the $p_{\perp}$ distribution are plotted in Fig.1b. We observe that the NLO corrections in the massless scheme are large and increase the cross section by roughly $100 \%$. In the massive scheme we have calculated only the LO result, which is between one and two orders of magnitude smaller than the LO massless cross section in the $p_{\perp}$ range between 3 and $15 \mathrm{GeV}$ NLO QCD corrections do not change this order-of-magnitude suppression [16] and will therefore not be taken into account for the resolved contribution in the massive scheme. We have examined that the prediction for the massless resolved cross section does not depend strongly on the particular choice of the NLO GRV parametrization of the $c$ distribution in the photon. The NLO GRV parametrization in the DIS $\gamma$ schemel yields similar results as well as the parametrization by Aurenche et al. [22].

\footnotetext{
${ }^{1}$ Similar results have been obtained in the LO analysis presented by R. Godbole at the Photon 95 conference, Sheffield, UK, April 1995 [21.

${ }^{2}$ To be consistent the appropriate changes have been made in the NLO direct part from the $\overline{\mathrm{MS}}$ to the $\mathrm{DIS}_{\gamma}$ scheme [17].
} 
We may ask where the strong contribution of the resolved cross section in the massless scheme is hidden in the massive approach. It must be contained in the $\ln \left(p_{\perp}^{2} / m^{2}\right)$ terms in the NLO direct cross section. In the NLO massless direct cross section all these terms are absorbed either in the structure function of the proton, the photon or the fragmentation function. Therefore at NLO the definition of direct/resolved photoproduction is ambiguous and only the sum of both contributions should be considered. In Fig.1c we have thus plotted the sum of the NLO massive direct and the LO massive resolved contribution from Figs.1a and b (denoted NLO/LO massive) and the same sum for the massless theory taken also from Figs.1a and b (called NLO/LO massless). Considering the same order in $\alpha_{s}$ these are the two predictions in the massive and massless theories which should be compared. We observe that the NLO/LO massless result is approximately $60 \%$ larger than the NLO/LO massive prediction. We have investigated this difference in some detail. To begin with one has to remember that in the massless theory the collinear singular contributions in the final charm states are absorbed into the fragmentation function. This subtraction is not performed in the massive theory. If we subtract the corresponding $\ln \left(p_{\perp}^{2} / m^{2}\right)$ terms in the massive theory the NLO/LO massive prediction is increased by $25 \%$, so that we are left still with a difference of $35 \%$. This difference originates mainly from the charm content of the photon which does not correspond exactly to the $\ln \left(p_{\perp}^{2} / m^{2}\right)$ terms in the massive theory arising from the almost collinear branching of photons into charm quark pairs. We have examined this further by substituting the GRV charm photon structure function by the massive LO "Bethe-Heitler" approximation [23]. This change reduces the difference between the massless and the massive NLO/LO predictions to a level of $10 \%$. Part of the difference between the two approaches can thus be attributed to theoretical uncertainties concerning the charm content of the photon. The remaining $\ln \left(p_{\perp}^{2} / m^{2}\right)$ terms connected with the collinear singularities at the proton leg are higher order in $\alpha_{s}$ and are definitely much less important.

In Fig.1c we have plotted also the sum of the direct and the resolved components for the massless theory including NLO corrections (denoted NLO massless). The higher order corrections to the resolved process increase the theoretical prediction by 35\% compared to the NLO/LO massless result. Following the discussion above this contribution should correspond to a large extent to the NNLO direct cross section in the massive scheme which has not been calculated yet.

In Fig.2 the differential cross sections $\mathrm{d}^{2} \sigma / \mathrm{d} y \mathrm{~d} p_{\perp}^{2}$ are shown for $p_{\perp}=10 \mathrm{GeV}$ as a function of the rapidity $y$ for the direct (Fig.2a) and the resolved contributions (Fig.2b). Comparing the results in the massive and the massless approaches we observe the same pattern as for the $p_{\perp}$ distribution, Figs.1a and b. From Fig.2a we can infer that both the LO and NLO predictions for the direct contribution in the massive and massless approaches are very close together. In Fig.2b, where the resolved $y$ differential cross sections are plotted we see the very much reduced cross section in the massive theory and the appreciable cross section in the massless approach, where the NLO prediction is in general larger than the LO result.

Fig.2c finally shows the sum of direct and resolved contributions in the massless and massive schemes. The same relation between massive and massless schemes is seen here as in the $p_{\perp}$ distribution in Fig.1c. 


\section{Conclusion}

We have observed that the cross section in the massless approach is approximately $110 \%$ larger than in the massive scheme. The main part of that deviation can be attributed to the difference found already in the comparison of the NLO/LO massless cross section with the NLO/LO massive result. This difference originates from several sources which have been disentangled. The remaining increase of the NLO massless prediction is due to the NLO corrections to the resolved process. Such a contribution corresponds mainly to a NNLO correction to the direct part which has not been calculated yet. In this sense the massless theory includes a significant part of the NNLO correction and should therefore yield a more reliable prediction than the massive scheme. This conclusion is supported by the observation that the massless prediction is much more stable under scale variation than the massive result.

Compared to a similar study for $b$ production in $p \bar{p}$ processes [18] we find much larger deviations between the massive and the massless approaches. This can be attributed to the much stronger influence of charm in the photon as compared to the proton caused by the point-like component of the photon structure function. Consequently a measurement of large- $p_{\perp}$ charm production at HERA might provide information about the charm content of the photon (cf. [21]). Such measurements will be very instructive since theoretical opinions on that issue are rather divided [24].

Our study was based on a scale-independent $\delta$-type fragmentation function of the charm quarks. In the future we shall extend this by using more realistic forms for the fragmentation including evolution to higher scales. This latter effect in the fragmentation might compensate part of the $\ln \left(p_{\perp}^{2} / m^{2}\right)$ absorption in the massless theory and might thus bring the massless prediction nearer to the massive result.

Note added: After completion of our work we have been informed about a similar analysis by Cacciari and Greco [25].

Acknowledgements. We thank the authors of 25] for making available to us their results prior to publication. One of us (BAK) is indebted to the FNAL Theory Group for inviting him as a Guest Scientist and for the great hospitality extended to him.

\section{References}

[1] M. Drees, R. Godbole, Phys. Rev. Lett. 61 (1988) 682.

[2] L.E. Gordon, J.K. Storrow, Phys. Lett. B291 (1992) 320.

[3] D. Bödeker, Phys. Lett. B292 (1992) 164.

[4] G. Kramer, S.G. Salesch, Z. Phys. C61 (1994) 320; D. Bödeker, G. Kramer, S.G. Salesch, Z. Phys. C63 (1994) 471.

[5] M. Derrick et al., ZEUS Collaboration, Phys. Lett. B297 (1992) 404, ibid. B 322 (1994) 287.

[6] T. Ahmed et al., H1 Collaboration, Phys. Lett. B297 (1992) 205.

[7] M. Derrick et al., ZEUS Collaboration, Phys. Lett. B342 (1995) 417. 
[8] M. Klasen, G. Kramer, S.G. Salesch, report DESY-94-232 (1994).

[9] F.M. Borzumati, B.A. Kniehl, G. Kramer, Z. Phys. C52 (1991) 13.

[10] J. Smith, W.K. Tung, preprint NIKHEF-H/93-20, published in Snowmass B Physics (1993).

[11] J.C. Collins, D.E. Soper, G. Sterman, Nucl. Phys. B263 (1986) 37.

[12] P. Nason, S. Dawson, R.K. Ellis, Nucl. Phys. B303 (1988) 607, ibid. B327 (1988) 49; R.K. Ellis, P. Nason, Nucl. Phys. B312 (1989) 551; W. Beenakker, H. Kuijf, W.L. van Neerven, J. Smith, Phys. Rev. D40 (1989) 54; J. Smith, W.L. van Neerven, Nucl. Phys. B374 (1992) 36; S. Frixione, M.L. Mangano, P. Nason, G. Ridolfi, Nucl. Phys. B412 (1994) 225.

[13] M. Drees, M. Krämer, J. Zunft, P.M. Zerwas, Phys. Lett. B306 (1993) 371.

[14] S. Frixione, M.L. Mangano, P. Nason, G. Ridolfi, Nucl. Phys. B431 (1994) 453;

M. Krämer, to appear in Proc. of "Photon '95", Sheffield, 1995.

[15] M. Derrick et al., ZEUS Collaboration, Phys. Lett. B349 (1995) 225.

[16] S. Frixione, M.L. Mangano, P. Nason, G. Ridolfi, Phys. Lett. B348 (1995) 633.

[17] B.A. Kniehl, G. Kramer, Z. Phys. C62 (1994) 53 and references therein.

[18] M. Cacciari, M. Greco, Nucl. Phys. B421 (1994) 530.

[19] A.D. Martin, R.G. Roberts, W.J. Stirling, report RAL-95-021 (1995).

[20] M. Glück, E. Reya, A. Vogt, Phys. Rev. D45 (1992) 3986, ibid. D46 (1992) 1973.

[21] M. Drees, R.M. Godbole, to appear in Proc. of "Photon '95", Sheffield, 1995.

[22] P. Aurenche, J.P. Guillet, M. Fontannaz, report ENSLAPP-A-435-93-REV (1994).

[23] E. Witten, Nucl. Phys. B104 (1976) 445; M. Glück, E. Reya, Phys. Lett. B83 (1979) 89.

[24] M.A.G. Aivazis, J.C. Collins, F.I. Olness, Wu-Ki Tung, Phys. Rev. D50 (1994) 3102; M. Glück, E. Reya, M. Stratmann, Nucl. Phys. B422 (1994) 37; F.I. Olness, S.T. Riemersma, preprint SMU-HEP/94/21 (1994).

[25] M. Cacciari, M. Greco, report FNT/T-95/12. 


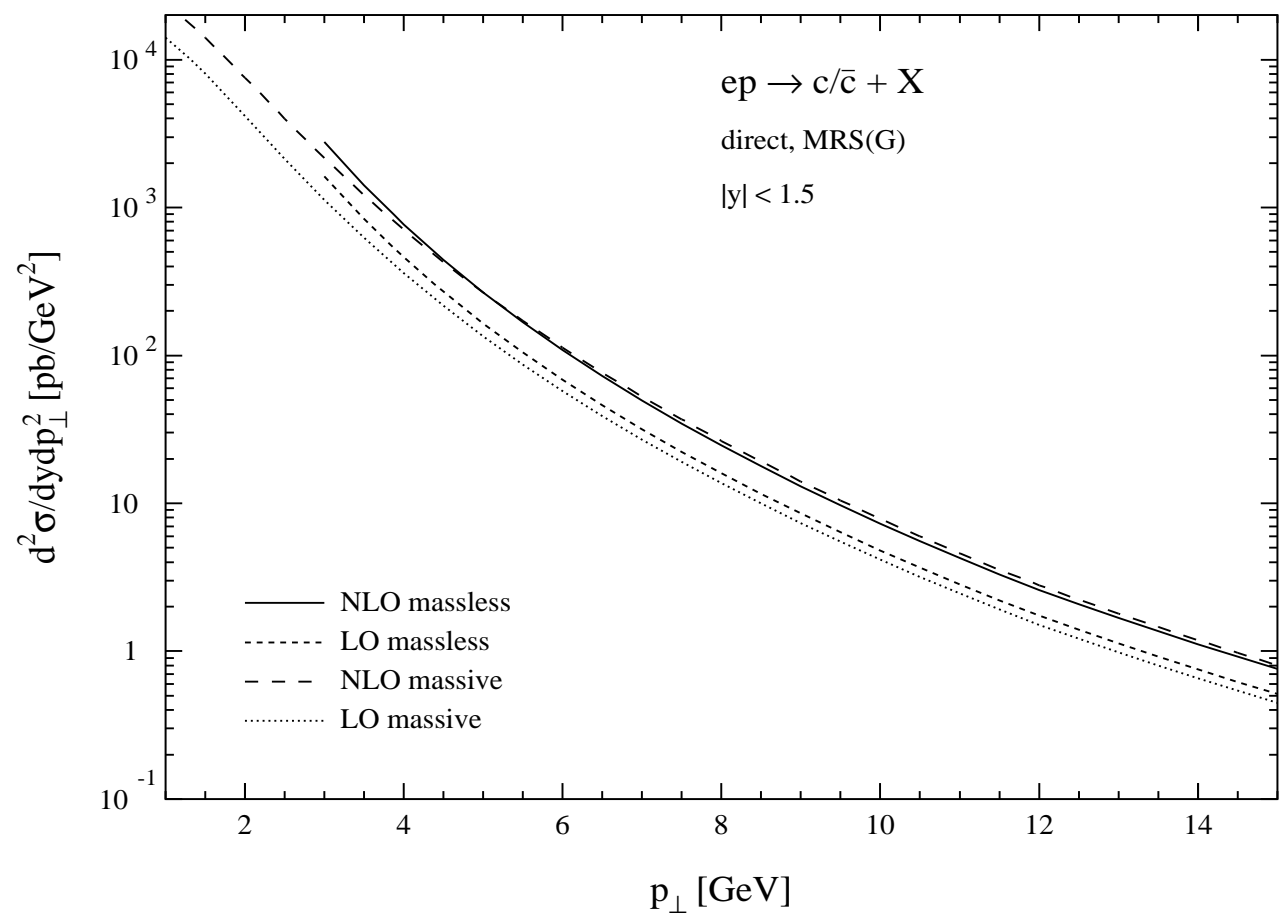

Fig.1a: Transverse momentum distributions of ep $\rightarrow c / \bar{c}+X$ averaged over the rapidity range $|y|<1.5$ for the direct contribution in the massless and massive schemes.

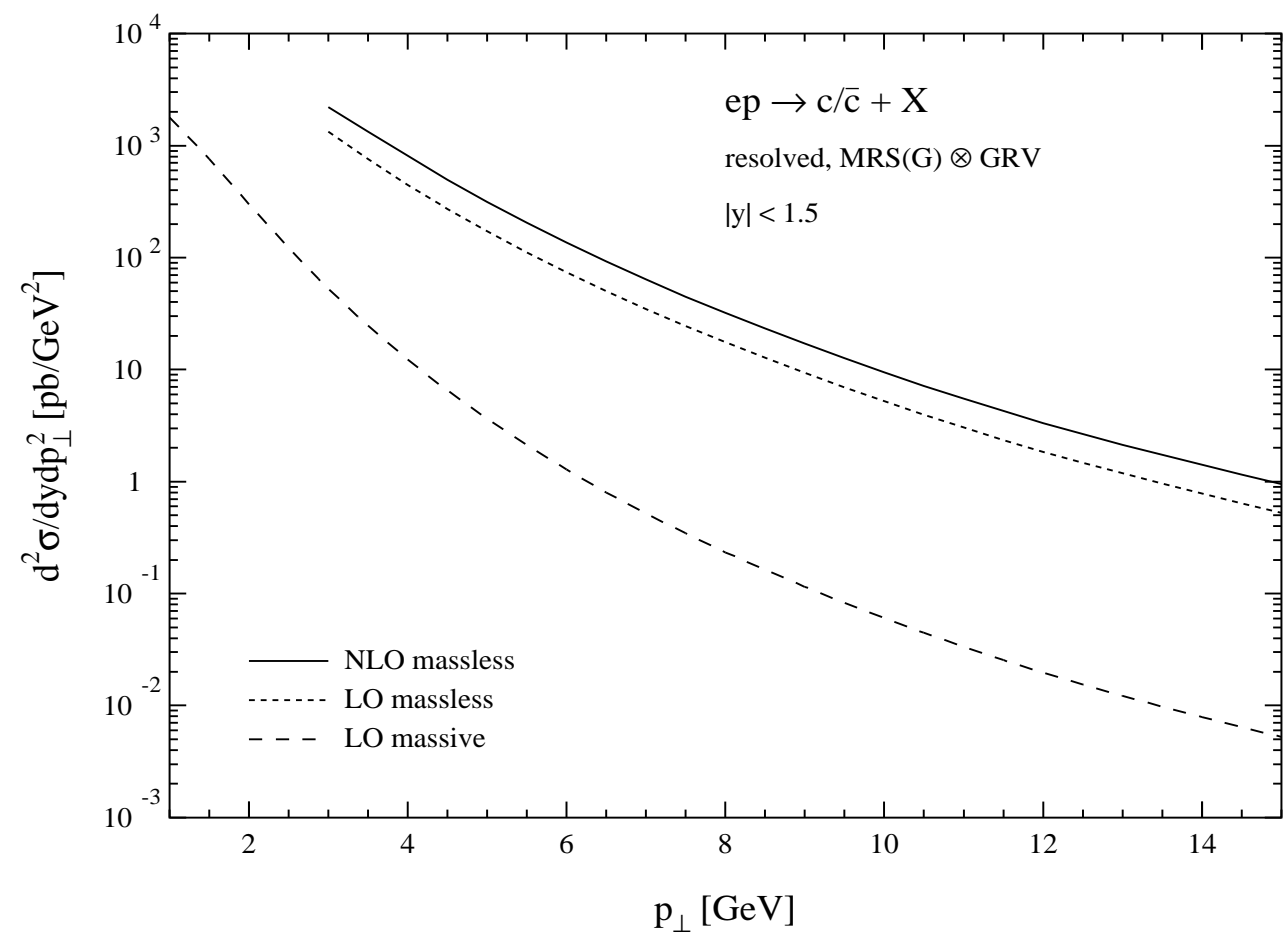

Fig.1b: Transverse momentum distributions of ep $\rightarrow c / \bar{c}+X$ averaged over the rapidity range $|y|<1.5$ for the resolved contribution in the massless and massive schemes. 


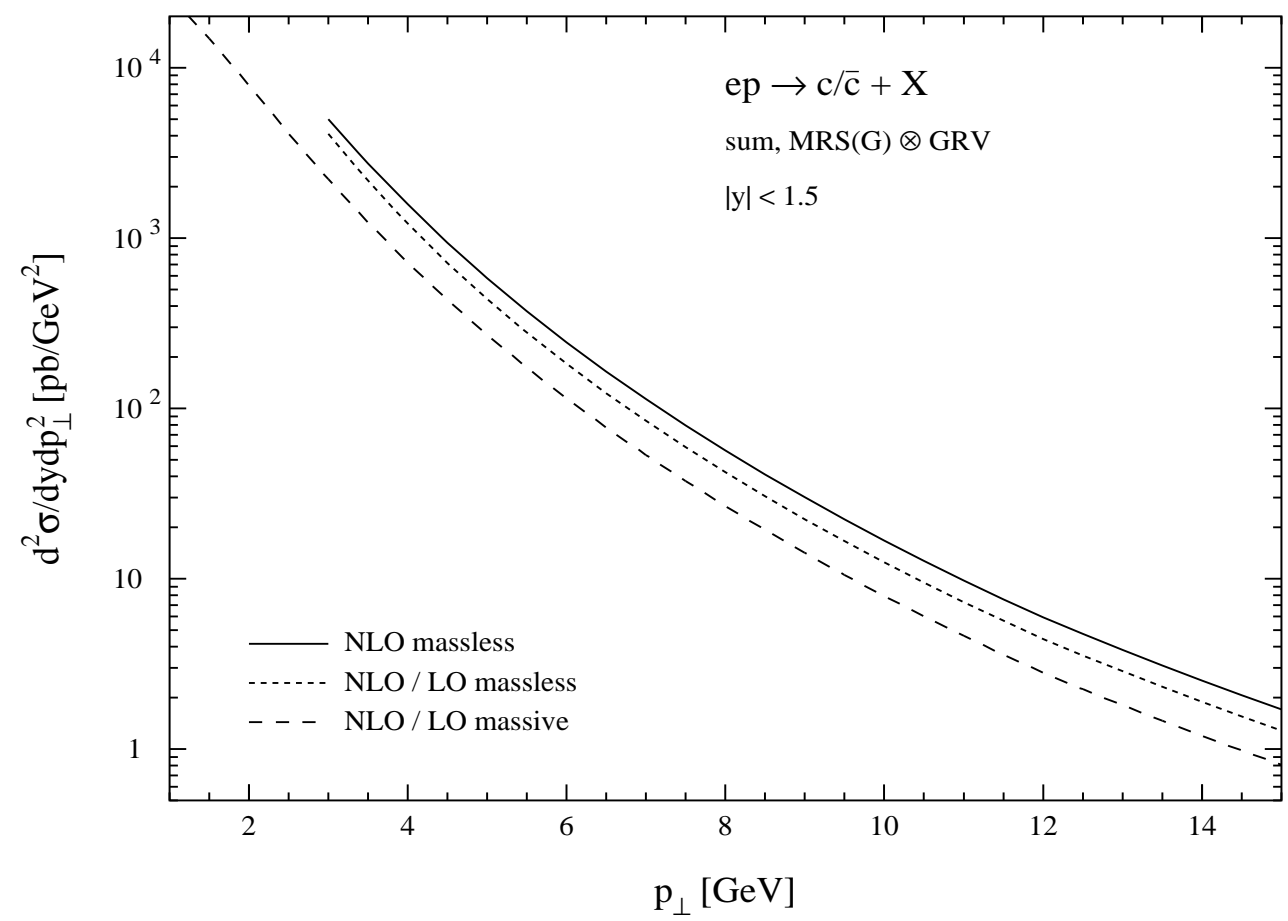

Fig.1c: Transverse momentum distributions of ep $\rightarrow c / \bar{c}+X$ averaged over the rapidity range $|y|<1.5$ in the massless and massive schemes. Shown is the sum of the NLO massive direct and the $L O$ massive resolved contributions (NLO/LO massive), the sum of the NLO massless direct and the $L O$ massless resolved contributions (NLO/LO massless), and the sum of the NLO massless direct and the NLO massless resolved contributions (NLO massless). 


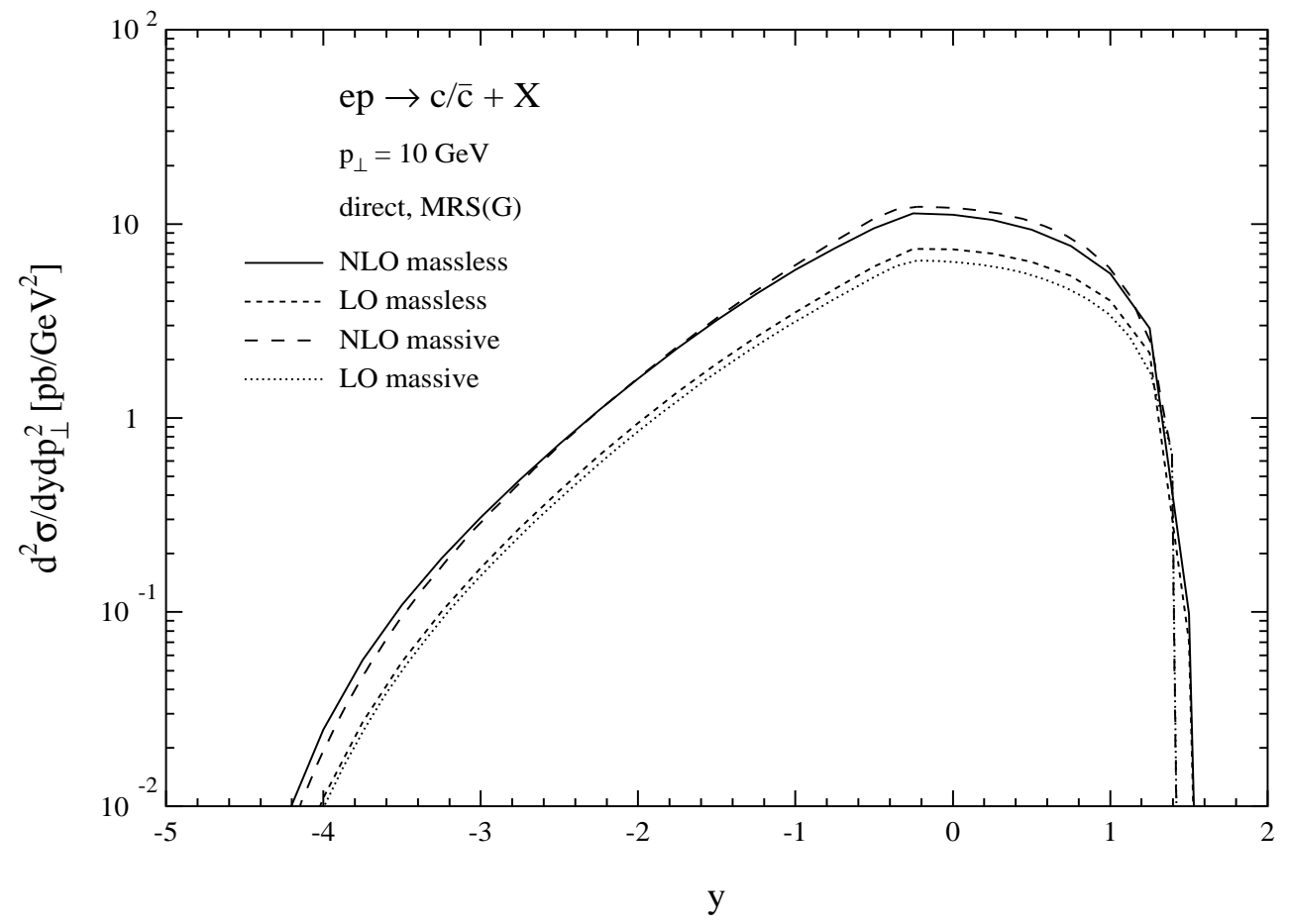

Fig.2a: Rapidity distributions of $e p \rightarrow c / \bar{c}+X$ at $p_{\perp}=10$ GeV for the direct contribution in the massless and massive schemes.

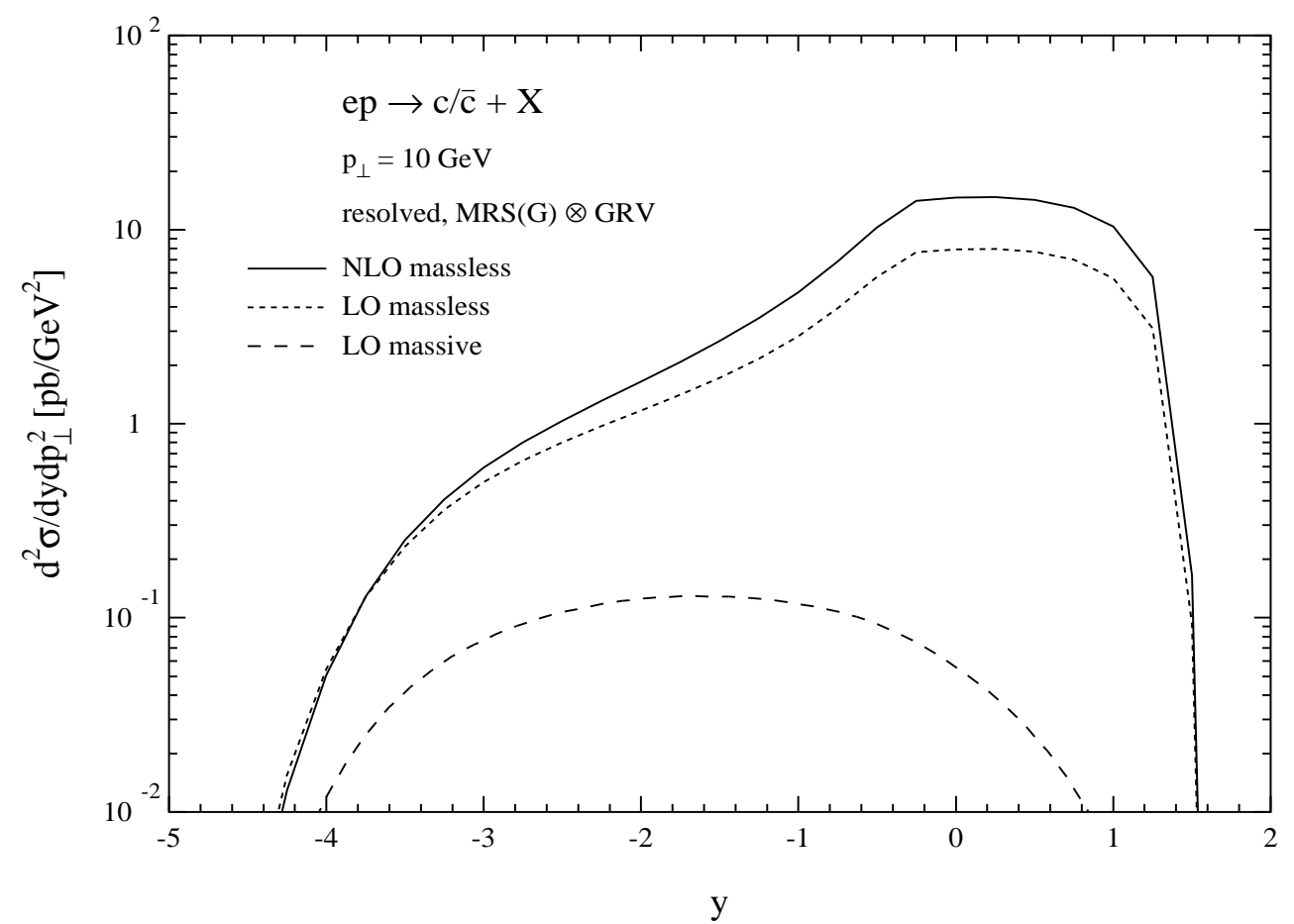

Fig.2b: Rapidity distributions of ep $\rightarrow c / \bar{c}+X$ at $p_{\perp}=10$ GeV for the resolved contribution in the massless and massive schemes. 


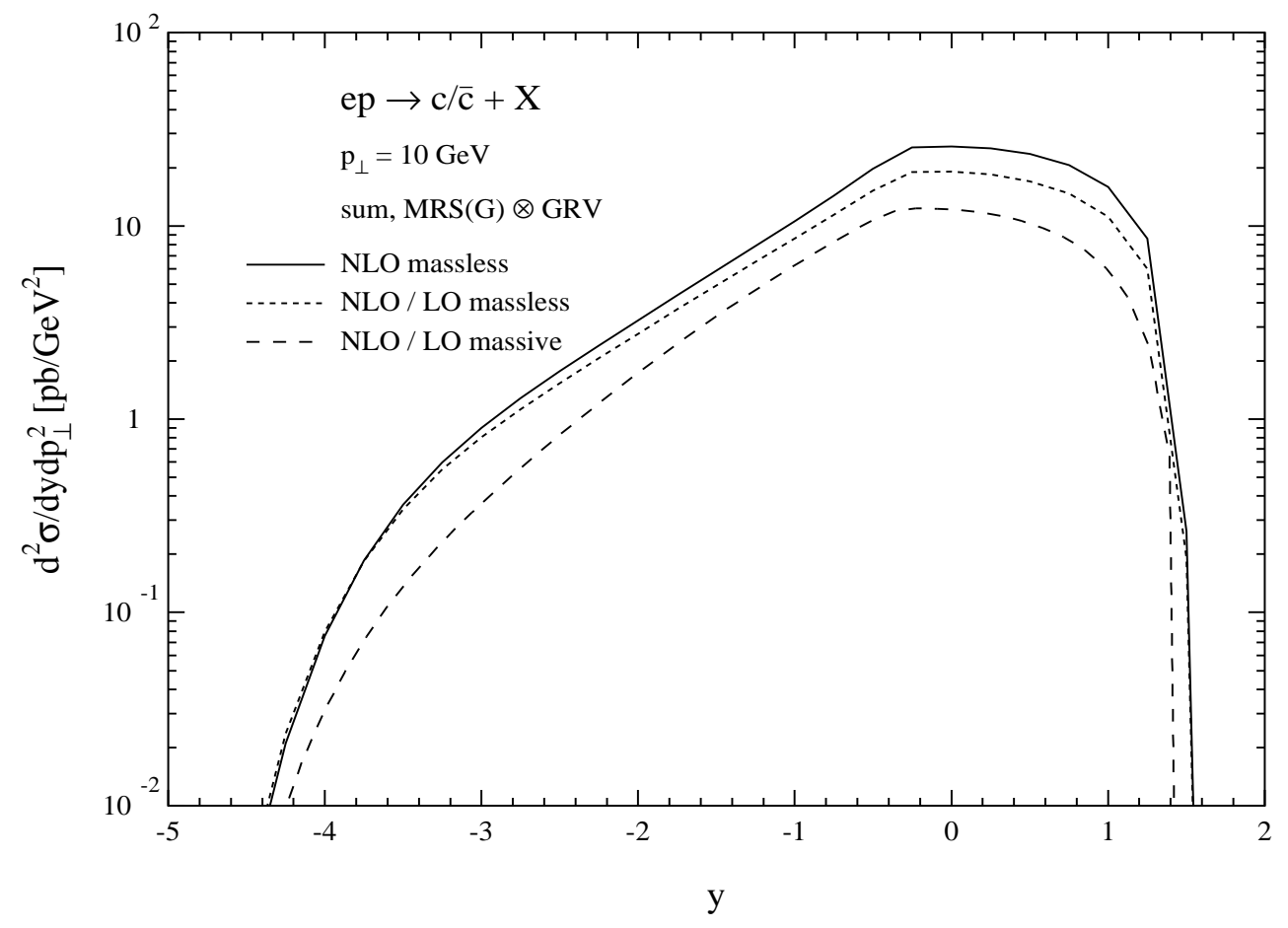

Fig.2c: Rapidity distributions of $e p \rightarrow c / \bar{c}+X$ at $p_{\perp}=10$ GeV for the resolved contribution in the massless and massive schemes. Shown is the sum of the NLO massive direct and the LO massive resolved contributions ( $N L O / L O$ massive), the sum of the NLO massless direct and the LO massless resolved contributions (NLO/LO massless), and the sum of the NLO massless direct and the NLO massless resolved contributions (NLO massless). 